\title{
Cost Benefit Analysis of Three Non-Identical Machine Model with Priority in Operation and Repair
}

\author{
Nafeesa Bashir* \\ Department of Statistics, University of Kashmir \\ nafeesabashir8@gmail.com \\ Raeesa Bashir \\ Mathematics and Quantitative Analysis, Amity University, Dubai, UAE \\ raisabashir52@gmail.com \\ J P Singh Joorel \\ Department of Statistics, University of Jammu \\ joorel@ rediffmail.com \\ T R Jan \\ Department of Statistics, University of Kashmir \\ drtrjan@gmail.com
}

Received: 15/Aug/2020

Revised: 10/May/2021

Accepted: 07/June/2021

\begin{abstract}
The paper proposes a new real life model and the main aim is to examine the cost benefit analysis of Textile Industry model subject to different failure and repair strategies. The reliability model comprises of three units i,e Spinning machine (S), Weaving machine $(\mathrm{W})$, Colouring and Finishing machine(Cf). The working principal of the model starts with spinning machine (S) where in unit $\mathrm{S}$ is in operative state while as weaving machine, Colouring and Finishing machine are in ideal state. Complete failure of system is observed when all three units of system i.e. S,W and Cf are in down state. Repairperson is always available to carry out the repair activities in the system in which first priority in repair is given to Colouring and Finishing machine followed by Spinning and weaving machine. The proposed model attempts to maximize the reliability of a real life system. Reliability measures such as Mean Sojourn time, Mean time to system failure, Profit analysis of system are examined to define the performance of the reliability characteristics. For concluding the study of such model, different stochastic measures are analyzed in steady state using regenerative point technique. The tables are prepared for arbitrary values of the parameters to show the performance of some important reliability measures and to check the efficiency of the model under such situations.
\end{abstract}

Keywords: Reliability Measures; Mean Sojourn Time; Laplace Transformation; Laplace -Stieltjes transformation.

\section{1- Introduction}

In the present scenario of global viable market, the basic objectives of industries is to reduce the production cost and enhance the productivity of manufacturing systems of processing Industries. To meet the increasing demand of the society, the industries must offer long term production. The efficiency and accessibility of industry can be made profitable in terms of higher production and lower maintenance costs. Availability of complex systems can be improved by considering different techniques of reliability like maintenance, inspection, repairs, priority to repair of the failed unit and so on. In addition, these techniques in industrial design can substantially strengthen operational dependability through better system design. A very trivial change in the technique of reliability can cause a profound change in the operational dependability, that is why it is vital to specify model reliability targets before any strategy plan is taken into consideration .

There are systems where upon failure in a complex system the repair of a component is given preference over the other component depending upon the significance of the component. Such systems are called priority systems. When a unit of lower priority is under repair and a unit of higher priority arrives for repair, the lower priority unit is pre-empted and higher priority unit is repaired. After completion of the service of higher priority unit, the lower higher priority unit is given service. In reliability modeling, it is found that accessibility and performance of failed and repairable systems can be enriched using redundancy technique. But due to the high cost, it is not always possible to keep the unit as spare. Also, it sometimes becomes essential to prioritize one unit over the other to increase performance, availability and hence the efficiency of the system. The idea of priority in repair discipline in reliability modeling has been 
considered by various authors researchers in order to make the system available for required service with least possible operational cost.

Kumar et al. [2] obtained reliability analysis of a warm standby model performing in normal/abnormal environment under certain assumptions where the distribution of failure time has been taken as negative exponential and the repair, inspection and replacement time distribution has been taken as arbitrary. Chander [11] discussed the two non-identical reliability models in which the failure and repair time of each unit were independent and uncorrelated random variables. The author obtained the various reliability measures using Regenerative Point Technique (R.P.T ). Also, Zhang Wang [15] studies a cold standby system with priority in use and repair. Malik et al. [12] studied cost- benefit analysis of a model under and gave priority to repair and degradation. The numerical and graphical behaviour of important reliability measures has been obtained for arbitrary values of costs and parameter. Further, Kadyan et al. [6] considered reliability performance of model under priority to repair and degradation. The authors obtained the availability and profit of the system using Regenerative point technique (RPT). Sureria and Malik [5], Malik [13] analyzed computer system with priority to software replacement over hardware replacement and obtained the reliability and economic measures of computer system under certain assumptions. The model has been analyzed stochastically by using R.P.T and the results of the model has been compared with the existing model. Chib et al. [3] assumed two types of repair facilities for stochastic analysis of two unit priority system. Rathee and Chander [9] carried out the reliability analysis of parallel system in which he gave priority to repair over maintenance. Munday and Malik[14] obtained profit analysis of a Computer System with Priority for hardware redundancy and repair. Recently Kumar et al. [4] studied profit analysis of computer systems with priority and maximum operation time. The author gave priority to software upgradation over hardware repair and replacement and hence obtained the profit of the system. The profit of the system has been compared with some existing model in literature. Also Bashir et al. [7] used the technique of inspection of degraded unit and obtained cost analysis of two unit model. Gorjian Jolfaei [8] carried out reliability analysis of power generation engines in a wastewater treatment plant. Kumar and Malik [1] examines the weathering server system with identical element in warm standby and used the concept of first come first serve repair policy for redundant systems in weather conditions. The various reliability measures of the system has been obtained using R.P.T. The author proved that the concept of first come first serve repair policy proved to be economically beneficial. Sharma and joorel [10] developed a model for fruit juice manufacturing system for its reliability analysis, which consists of four non identical units viz. Fruit cutter, Fruit pulper, Sterlizer and Homogenizer. The author used the concept of emergency repair in which the team of experts is available on the failure of Sterlizer or Homogenizer, to carry out the failure activities. [16] Obtained the reliability of two unit cold standby model under the assumption that the system is operating under different weather conditions and gave priority to preventive maintenance over inspection. To determine the nature of the system, various system effectiveness has been obtained using regenerative point technique and semi markov process. [17] Analysed the concrete mixture plant which comprises of seven units subject to the coverage factor by considering profust reliability approach which is based on probability assumption as well as fuzzy state assumption. The performance analysis of the system has been done using Markov birth-death model and differential equations solved by Runge-Kutta method. The results of the proposed model have proved beneficial for system designers. [18] have made an effort to obtain the system performance of sewage treatment plant by using markovian birth-death process. Various system measures like reliability, availability, maintainability and obtainability of the system has been obtained. [19] explored the generators in steam turbine power plant and obtained the availability and profit of generators. Using supplementary variable technique amd markov birth-death process different system measures has been evaluated. Results of the system have been obtained for availability and profit that would be useful for system designers to enrich the reliability and thus performance of plant

The basic aim of the paper is to obtain the profit evaluation of textile industry with exponential failure time distribution subject to different repair strategies. The System consists of three non-identical components Spinning (S), Weaving (W), Coloring and Finishing (Cf). Initially unit $\mathrm{S}$ is in working mode whereas unit $\mathrm{W}$ and $\mathrm{Cf}$ are in idle state. Failure of the system occurs only if all the unit fails. Single server is available in the system to carry out repair activities and $\mathrm{Cf}$ gets first priority over repair followed by $\mathrm{S}$ and $\mathrm{W}$. The failure time $(\mathrm{FT}) \sim \exp .(\eta)$. The repair time distribution is taken as general.

\section{2- Notations and Symbols}

- $\quad \eta_{1}, \eta_{2}, \eta_{3}=$ failure rates of unit $S, W, C f$ respectively.

- $\delta_{1}, \delta_{2}=$ activation rates of unit $W$ and $C f$ from idle to operation respectively

- $R(t), T(t), G(t)=$ cdf of repair time of the failed unit $S, W, C f$ respectively

- $* *=$ Laplace transform i.e. $\mathrm{f}^{*}(\mathrm{~s})=\int_{0}^{\infty} \mathrm{e}^{-\mathrm{st}} \mathrm{f}(\mathrm{t}) \mathrm{dt}$ 
TRANSITION DIAGRAM

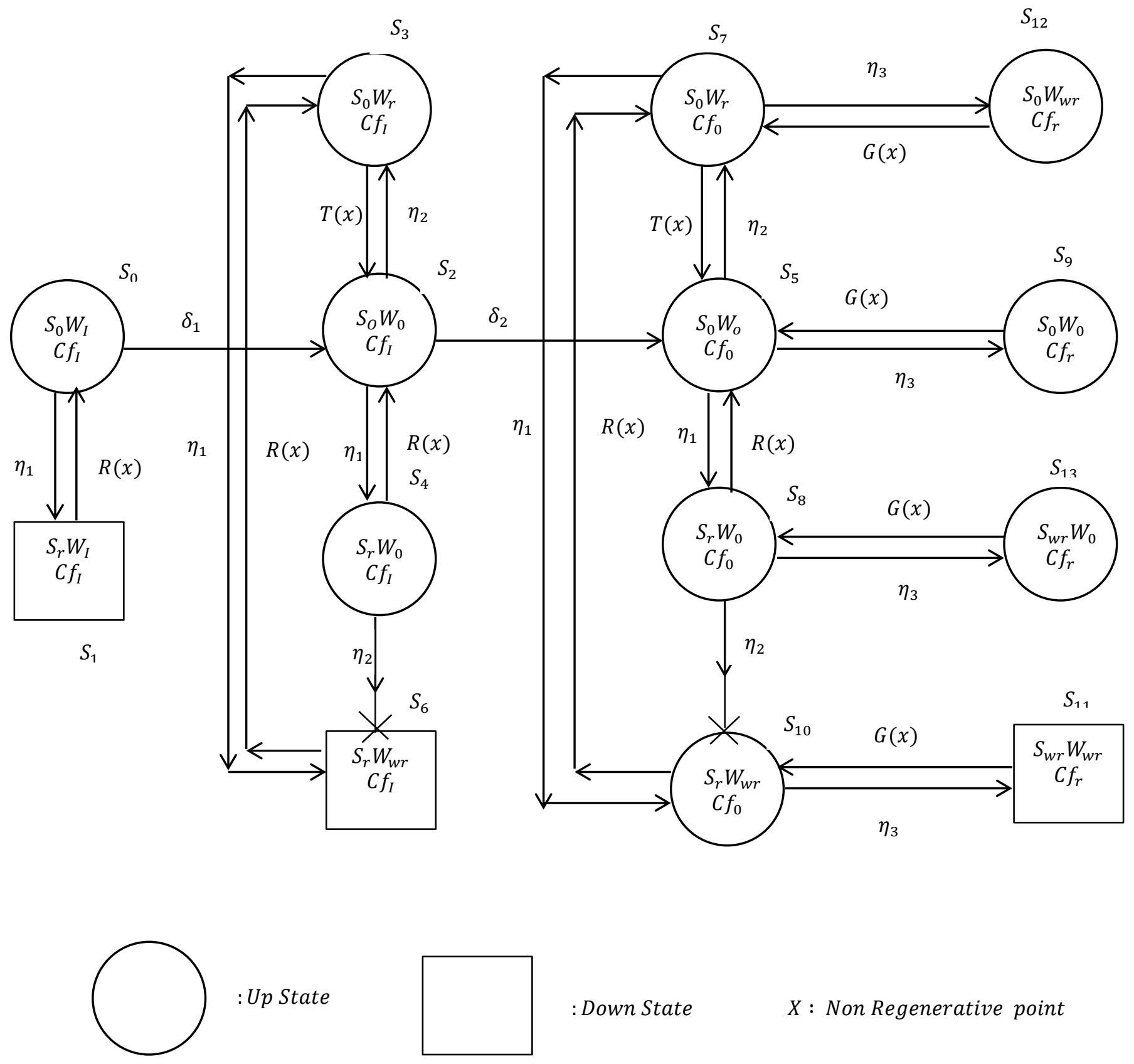




$$
\begin{aligned}
& \sim=\text { Laplace-Stieltjes transform i.e. } \tilde{\mathrm{F}}(\mathrm{s})= \\
& \int_{0}^{\infty} \mathrm{e}^{-\mathrm{st}} \mathrm{dF}(\mathrm{t}) .
\end{aligned}
$$

- $\quad S_{0}, W_{0}, C f_{0}=S, W, C f$ are normal and operative.

- $S_{r}, W_{r}, C f_{r}=S, W, C f$ are under repair.

- $S_{w r}, W_{w r}, C f_{w r}=S, W, C f$ are waiting for repair.

- $W_{I}, C f_{I}=W, C f$ is in ideal state.

- $S_{0}=$ Total possible states of the system, $i=$ $0,1,2,3 \ldots 13$

$$
\begin{aligned}
S_{0} & =\left[S_{0}, W_{I}, C f_{I}\right] \\
S_{1} & =\left[S_{r}, W_{I}, C f_{I}\right] \\
S_{2} & =\left[S_{0}, W_{0}, C f_{I}\right] \\
S_{3} & =\left[S_{0}, W_{r}, C f_{I}\right] \\
S_{4} & =\left[S_{r}, W_{0}, C f_{I}\right] \\
S_{5} & =\left[S_{0}, W_{0}, C f_{0}\right] \\
S_{6} & =\left[S_{r}, W_{w r}, C f_{I}\right] \\
S_{7} & =\left[S_{0}, W_{r}, C f_{0}\right] \\
S_{8} & =\left[S_{r}, W_{0}, C f_{0}\right] \\
S_{9} & =\left[S_{0}, W_{0}, C f_{r}\right] \\
S_{10} & =\left[S_{r}, W_{w r}, C f_{0}\right] \\
S_{11} & =\left[S_{w r}, W_{w r}, C f_{r}\right] \\
S_{12} & =\left[S_{0}, W_{w r}, C f_{r}\right] \\
S_{13}= & {\left[S_{w r}, W_{0}, C f_{r}\right] }
\end{aligned}
$$

\section{3- Transition Probabilities}

Let $Q_{i j}(t)$ represents the transition probability from state $i$ to $j$ in transient state. Then the various transition probabilities, can be written as follows:

$$
\begin{aligned}
& Q_{01}(t)=\eta_{1} \int_{0}^{t} e^{-\left(\eta_{1}+\delta_{1}\right) u} d u \\
& Q_{02}(t)=\delta_{1} \int_{0}^{t} e^{-\left(\eta_{1}+\delta_{1}\right) u} d u \\
& Q_{10}(t)=R(u) d u \\
& Q_{23}(t)=\eta_{2} \int_{0}^{t} e^{-\left(\eta_{1}+\eta_{2}+\delta_{2}\right) u} d u \\
& Q_{24}(t)=\eta_{1} \int_{0}^{t} e^{-\left(\eta_{1}+\eta_{2}+\delta_{2}\right) u} d u
\end{aligned}
$$

$$
\begin{aligned}
& Q_{25}(t)=\delta_{2} \int_{0}^{t} e^{-\left(\eta_{1}+\eta_{2}+\delta_{2}\right) u} d u \\
& Q_{32}(t)=\int_{0}^{t} e^{-\left(\eta_{1}\right) u} d t u \\
& Q_{36}(t)=\eta_{1} \int_{0}^{t} e^{-\left(\eta_{1}\right) u} \bar{T}(u) d u \\
& Q_{42}(t)=\int_{0}^{t} e^{-\left(\eta_{2}\right) u} d r(u) \\
& Q_{57}(t)=\eta_{2} \int_{0}^{t} e^{-\left(\eta_{1}+\eta_{2}+\eta_{3}\right) u} d u \\
& Q_{58}(t)=\eta_{1} \int_{0}^{t} e^{-\left(\eta_{1}+\eta_{2}+\eta_{3}\right) u} d u \\
& Q_{59}(t)=\eta_{3} \int_{0}^{t} e^{-\left(\eta_{1}+\eta_{2}+\eta_{3}\right) u} d u \\
& Q_{63}(t)=R(u) d u \\
& Q_{75}(t)=\int_{0}^{t} e^{-\left(\eta_{2}+\eta_{3}\right) u} d u \\
& Q_{7,10}(t)=\eta_{1} \int_{t}^{t} e^{-\left(\eta_{1}+\eta_{3}\right) u} \bar{T}(u) d u \\
& Q_{7,12}(t)=\eta_{3} \int_{0}^{t} e^{-\left(\eta_{1}+\eta_{3}\right) u} \bar{T}(u) d u \\
& Q_{8,13}(t)=\eta_{3} \int_{0}^{t} e^{-\left(\eta_{2}+\eta_{3}\right) u} \bar{R}(u) d u \\
& Q_{8,11}(t)=\eta_{2} \eta_{3} \int_{0}^{t} \int_{0}^{v} e^{-\left(\eta_{2}\right) u} d u e^{-\left(\eta_{3}\right) v} \bar{R}(v) d v \\
& Q_{85}(t)=\int_{0}^{t} e^{-\left(\eta_{2}+\eta_{3}\right) u} d R(u) \\
& Q_{87}(t)=\eta_{2} \int_{0}^{t} \int_{0}^{v} e^{-\left(\eta_{2}\right) u} d u e^{-\left(\eta_{3}\right) v} d R(v) d v \\
& Q_{95}(t)=\int_{0}^{t} G(u) d u \\
& Q_{10,7}(t)=\int_{0}^{t} e^{-\left(\eta_{3}\right) u} d R(u) \\
& Q_{10,11}(t)=\int_{0}^{t} e^{-\left(\eta_{3}\right) u} \bar{R}(u) d u \\
& Q_{11,10}(t)=Q_{12,7}(t)=Q_{13,8}(t)=\int_{0}^{t} G(u) d u
\end{aligned}
$$

In transition probabilities by taking $t \rightarrow \infty$ we get the steady state probabilities i.e $p_{i j}=Q_{i j}(\infty)=\int_{0}^{t} Q_{i j}(t)$ and 
it can be easily verified that $\sum_{j} p_{i j}=1$ for all possible values of $i$, i.e

$$
\begin{aligned}
& p_{01}+p_{02}=1 \\
& p_{10}=1 \\
& p_{23}+p_{24}+p_{25}=1 \\
& p_{32}+p_{36}=1 \\
& p_{42}+p_{43}^{(6)}=1 \\
& p_{57}+p_{58}+p_{59}=1 \\
& p_{63}=1 \\
& p_{75}+p_{7,10}+p_{7,12}=1 \\
& p_{85}+p_{87}^{(10)}+p_{87}^{(11)}+p_{8,13}=1 \\
& p_{95}=1 \\
& p_{10,7}+p_{10,11}=1 \\
& p_{11,10}=p_{12,7}=p_{13,8}=1
\end{aligned}
$$

\subsection{Mean Sojourn Times(MST)}

The MST $\phi_{i}$ is the expected time taken by a system in a given state before transiting to any other state. If $T_{i}$ is the sojourn time of $S_{i}$ then MST $\phi_{i}$ is given by:

$$
\begin{aligned}
& \phi_{i}=\int_{0}^{\infty} \mathrm{P}\left(\mathrm{T}_{\mathrm{i}}>t\right) \mathrm{dt} \\
& \phi_{0}=\int_{0}^{t} e^{-\left(\eta_{1}+\delta_{1}\right) t} d t=\frac{1}{\eta_{1}+\delta_{1}} \\
& \phi_{1}=\int_{0}^{\infty} \bar{R}(t) d t \\
& \phi_{2}=\int_{0}^{\infty} e^{-\left(\eta_{1}+\eta_{2}+\delta_{2}\right) t} d t=\frac{1}{\eta_{1}+\eta_{2}+\delta_{2}} \\
& \phi_{3}=\int_{0}^{\infty} e^{-\left(\eta_{1}\right) t} \bar{T}(t) d t=\frac{1}{\eta_{1}}\left[1-\tilde{T}\left(\eta_{1}\right)\right] \\
& \phi_{4}=\int_{0}^{\infty} e^{-\left(\eta_{2}\right) t} \bar{R}(t) d t=\frac{1}{\eta_{2}}\left[1-\tilde{R}\left(\eta_{1}\right)\right] \\
& \phi_{5}=\int_{0}^{\infty} e^{-\left(\eta_{1}+\eta_{2}+\eta_{3}\right) t} d t \\
& \phi_{6}=\int_{0}^{\infty} \bar{R}(t) d t
\end{aligned}
$$

$$
\begin{aligned}
& \phi_{7}=\int_{0}^{\infty} e^{-\left(\eta_{2}+\eta_{3}\right) t} \bar{T}(t) d t=\frac{1}{\eta_{2}+\eta_{3}}\left[1-\tilde{T}\left(\eta_{2}+\eta_{3}\right)\right] \\
& \phi_{8}=\int_{0}^{\infty} e^{-\left(\eta_{2}+\eta_{3}\right) t} \bar{R}(t) d t=\frac{1}{\eta_{2}+\eta_{3}}\left[1-\tilde{R}\left(\eta_{2}+\eta_{3}\right)\right] \\
& \phi_{9}=\phi_{11}=\phi_{12}=\phi_{13}=\int_{0}^{\infty} \bar{G}(t) d t \\
& \phi_{10}=\int_{0}^{\infty} e^{-\left(\eta_{3}\right) t} \bar{R}(t) d t=\frac{1}{\eta_{3}}\left[1-\tilde{R}\left(\eta_{3}\right)\right]
\end{aligned}
$$

\section{4- Mean Time to System Failure}

We define $\mathrm{T}_{\mathrm{i}}$ as the time to system failure and $\pi_{i}(t)$ be the c.d.f. of the time to system failure for the first time when the system starts operation from state $S_{i}$. We find the equations of $\pi_{i}(t)$ for various values of $i$, by using regenerative point technique.

Therefore, MTSF for different states is given by:

$$
\begin{aligned}
& \pi_{0}(t)=Q_{01}(t)+Q_{02}(t) \circledast \pi_{2}(t) \\
& \pi_{2}(t)=Q_{23}(t) \circledast \pi_{3}(t)+Q_{24}(t) \circledast \pi_{4}(t)+Q_{25}(t) \circledast \pi_{5}(t) \\
& \pi_{3}(t)=Q_{32}(t) \circledast \pi_{2}(t)+Q_{36}(t) \\
& \pi_{4}(t)=Q_{42}(t) \circledast \pi_{2}(t)+Q_{46}(t) \\
& \pi_{5}(t)=Q_{57}(t) \circledast \pi_{7}(t)+Q_{58}(t) \circledast \pi_{8}(t)+Q_{59}(t) \circledast \pi_{9}(t) \\
& \pi_{7}(t)=Q_{75}(t) \circledR \pi_{5}(t)+Q_{7,10}(t) \circledR \pi_{10}(t) \\
& +Q_{7,12}(t) \circledR \pi_{12}(t) \\
& \pi_{8}(t)=Q_{85}(t) \circledast \pi_{5}(t)+Q_{8,10}(t) \circledast \pi_{10}(t)+ \\
& Q_{8,13}(t) \circledast \pi_{13}(t) \\
& \pi_{9}(t)=Q_{95}(t) \circledast \pi_{5}(t) \\
& \pi_{10}(t)=Q_{10,7}(t) \circledast \pi_{7}(t)+Q_{10,11}(t) \\
& \pi_{12}(t)=Q_{12,7}(t) \circledast \pi_{7}(t) \\
& \pi_{13}(t)=Q_{13,8}(t) \circledR \pi_{8}(t)
\end{aligned}
$$

Taking L.S.T of above equations, and simplifying for $\tilde{\pi}_{0}(s)$

$\tilde{\pi}_{0}(s)=\frac{N_{1}(s)}{D_{1}(s)}$

On taking $\mathrm{s} \rightarrow 0$ in above equation and $\widetilde{\mathrm{Q}}_{\mathrm{ij}}(\mathrm{s}) \rightarrow \mathrm{p}_{\mathrm{ij}}$, as $s \rightarrow 0$, we have

$\tilde{\pi}_{0}(s)=\frac{N_{1}(s)}{D_{1}(s)}=1$ implies that $\pi_{0}(t)$ is a proper cdf.

Therefore, MTSF is given by 


$$
E(T)=-\left.\frac{d \widetilde{\pi}_{0}(s)}{d s}\right|_{s=0}=\frac{D_{1}^{\prime}(0)-N_{1}^{\prime}(0)}{D_{1}(0)}
$$

Where,

$$
\begin{aligned}
& D^{\prime}{ }_{1}(0)-N^{\prime}{ }_{1}(0)= \\
& {\left[\phi_{0}\left\{1-p_{23} p_{32}-p_{24} p_{42}\right\}+\phi_{2} p_{02}+\phi_{3} p_{23} p_{02}+\right.} \\
& \left.\phi_{4} p_{02} p_{24}\right]+\left[\left(1-p_{7,10} p_{10,7}-p_{7,12}\right)\right]\left[\left(1-p_{8,13}\right)-\right. \\
& \left.\left\{p_{58} p_{85}+p_{59}\left(1-p_{8,13}\right)\right\}\right]-\left[p _ { 7 5 } \left(p_{57}\left(1-p_{8,13}\right)+\right.\right. \\
& \left.\left.p_{58} p_{8,10} p_{10,7}\right)\right]+\phi_{5} p_{75}\left(1-p_{8,13}\right)\left(1-p_{23} p_{32}-\right. \\
& \left.p_{24} p_{42}\right)+\phi_{7}\left\{p_{57}\left(1-p_{8,13}\right)+p_{58} p_{8,10} p_{10,7}\right\}(1- \\
& \left.p_{23} p_{32}-p_{24} p_{42}\right)+\phi_{8} p_{58}\left(1-p_{7,10} p_{10,7}-p_{7,12}\right)(1- \\
& \left.p_{23} p_{32}-p_{24} p_{42}\right)+\phi_{9} p_{59}\left(1-p_{8,13}\right)\left(1-p_{7,10} p_{10,7}-\right. \\
& \left.p_{7,12}\right)\left(1-p_{23} p_{32}-p_{24} p_{42}\right)+\phi_{10}\left[p _ { 7 , 1 0 } \left[\left(1-p_{8,13}\right)-\right.\right. \\
& \left.\left.\left\{p_{58} p_{85}+p_{59}\left(1-p_{8,13}\right)\right\}\right]+p_{58} p_{8,10}\right] \quad\left(1-p_{23} p_{32}-\right. \\
& \left.p_{24} p_{42}\right)+\phi_{12} p_{7,12}\left[\left(1-p_{8,13}\right)-\left\{p_{58} p_{85}+p_{59}(1-\right.\right. \\
& \left.\left.\left.p_{8,13}\right)\right\}\right]\left(1-p_{23} p_{32}-p_{24} p_{42}\right)+\phi_{13}\left[\left(p_{8,13}-\right.\right. \\
& \left.\left.p_{59} p_{8,13}\right)\left(1-p_{7,10} p_{10,7}-p_{7,12}\right)-p_{57} p_{75} p_{8,13}\right](1- \\
& \left.p_{23} p_{32}-p_{24} p_{42}\right) \\
& D_{1}(0)=\left(1-p_{23} p_{32}-p_{24} p_{42}\right)\left[\left\{1-p_{7,10} p_{10,7}-\right.\right. \\
& \left.p_{7,12} p_{12,7}\right\}\left\{\left(1-p_{8,13} p_{13,8}\right)-\left\{p_{58} p_{85}+p_{59}(1-\right.\right. \\
& \left.\left.\left.\left.p_{8,13}\right)\right\}\right\}-\left\{p_{75}\left(p_{57}\left(1-p_{8,13}\right)+p_{58} p_{8,10} p_{10,7}\right)\right\}\right]
\end{aligned}
$$

\section{5- Availability Analysis}

We define availability, $A_{i}(t)$ as the probability that the system is in upstate and is available to serve its required purpose when it initially start from regenerative state $S_{I}$ The availability equations for different states is given as:

$$
\begin{aligned}
& A_{0}(t)=M_{0}(t)+q_{01}(t)\left(C A_{1}(t)+q_{02}(t)(C) A_{2}(t)\right. \\
& A_{1}(t)=q_{10}(t) \Subset A_{0}(t) \\
& A_{2}(t)=M_{2}(t)+q_{23}(t)(C) A_{3}(t)+q_{24}(t)(C) A_{4}(t) \\
& +q_{24}(t) \subseteq A_{4}(t) \\
& A_{3}(t)=M_{3}(t)+q_{32}(t)\left(\mathbb{C} A_{2}(t)+q_{36}(t)\left(\mathbb{C} A_{6}(t)\right.\right. \\
& A_{4}(t)=M_{4}(t)+q_{42}(t) \text { (C) } A_{2}(t)+q_{43}^{(6)}(t) \text { (C) } A_{3}(t) \\
& A_{5}(t)=M_{0}(t)+q_{01}(t) \mathbb{C} A_{1}(t)+q_{02}(t) \mathbb{C} A_{2}(t) \\
& A_{6}(t)=q_{63}(t) \subseteq A_{3}(t) \\
& A_{7}(t)=M_{7}(t)+q_{75}(t)\left(A_{5}(t)+q_{7,10}(t)(C) A_{10}(t)\right. \\
& +q_{7,12}(t)\left(A_{12}(t)\right. \\
& A_{8}(t)=M_{8}(t)+q_{85}(t) \Subset A_{5}(t)+q_{87}^{(10)}(t) \Subset A_{7}(t) \\
& +q_{8,11}^{(10)}(t) \Subset A_{11}(t)+q_{8,13}(t) \Subset A_{13}(t) \\
& A_{9}(t)=M_{9}(t)+q_{95}(t)\left(\mathbb{C} A_{5}(t)\right. \\
& A_{10}(t)=M_{10}(t)+q_{10,7}(t) \subseteq A_{7}(t)+ \\
& q_{10,11}(t) \subseteq A_{11}(t)
\end{aligned}
$$

$$
\begin{aligned}
& A_{11}(t)=q_{11,10}(t)\left(A_{10}(t)\right. \\
& A_{12}(t)=M_{12}(t)+q_{12,7}(t) \text { (C) } A_{7}(t) \\
& A_{13}(t)=M_{13}(t)+q_{13,8}(t) \text { C } A_{8}(t)
\end{aligned}
$$

Taking the LT of above equations, we get the set of linear equations in $A_{i}^{*}(s)$ and solving for $A_{0}^{*}(s)$, we get

$$
A_{0}^{*}(s)=\frac{N_{2}(s)}{D_{2}(s)}
$$

The steady state availability is given as:

$\mathrm{A}_{0}=\lim _{\mathrm{s} \rightarrow 0} \mathrm{~s} \mathrm{~A} \mathrm{~A}_{0}^{*}(\mathrm{~s})=N_{2}(0) / D_{2}^{\prime}(0)$

where,

$N_{2}(0)=\left[\left\{p_{32}\left(1-p_{23}\right)-p_{24} p_{32}\right\} \phi_{0}+\left\{p_{02}\left(\left(\phi_{2}+\right.\right.\right.\right.$

$\left.\left.\left.\left.p_{24} \phi_{4}\right) p_{32}+\left(p_{23} p_{24} p_{43}\right)\right)\right\}\right]\left[\left\{p_{10,7}-p_{7,10} p_{7,12}\right\}\{(1-\right.$

$\left.\left.p_{8,13}\right)\left(1-p_{59}\right)-p_{58} p_{85}\right\}-\left\{p_{57}\left(1-p_{8,13}\right)+\right.$

$\left.\left.p_{58} p_{87}^{(10)}\right\}\left(p_{75} p_{10,7}\right)\right]+p_{02} p_{25}\left[\left\{\left(\phi_{5}+p_{59} \phi_{9}\right)(1-\right.\right.$

$\left.\left.p_{8,13}\right)+p_{58}\left(\phi_{8}+p_{8,13} \phi_{13}\right)\right\}\left(p_{10,7}\left(1-p_{7,12}\right)\right)+$

$\left\{p_{57}\left(1-p_{8,13}\right)+p_{58} \mathrm{p}_{87}^{(10)}\right\}\left\{\left(\phi_{7}+p_{7,12} \phi_{12}\right)(1-\right.$

$\left.\left.\left.p_{7,10}\right) p_{10,7}+p_{7,10}\left\{\phi_{10}+p_{10,7}\left(\phi_{7}+p_{7,12} \phi_{12}\right)\right\}\right\}\right]$

$D_{2}^{\prime}(0)=\left[\phi_{5}\left(1-p_{8,13}\right)+\phi_{8} p_{58}\right] p_{02} p_{25} p_{32} p_{75} p_{10,7}+$ $\left[\phi_{7}\left\{p_{57}\left(1-p_{8,13}\right)+p_{58} p_{87}^{(10)}\right\}+\phi_{9} p_{59} p_{75}(1-\right.$

$\left.\left.p_{8,13}\right)\right] p_{02} p_{25} p_{32} p_{10,7}+\phi_{10}\left[\left(1-p_{7,12}\right)\left\{\left(1-p_{8,13}\right)(1-\right.\right.$

$\left.\left.\left.p_{59}\right)-p_{58} p_{85}\right\}-p_{75}\left\{p_{57}\left(1-p_{8,13}\right)+p_{58} p_{87}^{(10)}\right\}\right]$

$p_{02} p_{32} p_{25}+\phi_{11}\left[p_{10,11}\left(1-p_{7,12}\right)\left\{\left(1-p_{8,13}\right)(1-\right.\right.$

$\left.\left.p_{59}\right)-p_{58} p_{85}\right\}-p_{75} p_{10,11}\left\{p_{57}\left(1-p_{8,13}\right)+\right.$

$\left.\left.p_{58} p_{87}^{(10)}\right\}\right] p_{02} p_{32} p_{25}+\left[\phi_{12}\left\{\left(1-p_{8,13}\right)\left(1-p_{59}\right)-\right.\right.$

$\left.\left.p_{58} p_{85}\right\} p_{7,12}+\phi_{13} p_{75} p_{8.13} p_{58}\right] p_{10,7} p_{02} p_{25} p_{32}$

\section{6- Busy Period Analysis (BPA)}

It is the probability that the repairman is occupied in repairing the failed component at time ' $t$ ' given that the system has started from regenerative state $S_{i}$ at $t=0$. The BPA for different states is given by

$$
\begin{aligned}
& B_{0}(t)=q_{01}(t) \Subset B_{1}(t)+q_{02}(t) \Subset B_{2} \\
& B_{1}(t)=M_{1}+q_{10}(t) \Subset B_{0}(t) \\
& B_{2}(t)=q_{23}(t) \Subset B_{3}(t)+q_{24}(t) \Subset B_{4}+q_{25}(t) \Subset B_{5} \\
& B_{3}(t)=M_{3}+q_{32}(t) \Subset B_{2}(t)+q_{36}(t) \Subset B_{6}(t) \\
& B_{4}(t)=M_{4}+q_{42}(t) @ B_{2}(t)+q_{43}^{(6)}(t) \Subset B_{3}(t)
\end{aligned}
$$




$$
\begin{aligned}
& B_{5}(t)=M_{5}+q_{57}(t) \Subset B_{7}(t)+q_{58}(t) \Subset B_{8}(t)+ \\
& q_{59}(t) \subset B_{9}(t) \\
& B_{6}(t)=M_{6}+q_{63}(t) \subseteq B_{3}(t) \\
& B_{7}(t)=M_{7}+q_{75}(t) \Subset B_{5}(t)+q_{7,10}(t) @ B_{10}(t)+ \\
& q_{7,12}(t)\left(B_{12}\right. \\
& B_{8}(t)=M_{8}+q_{85}(t) \Subset B_{5}(t)+q_{87}^{(10)}(t) \Subset B_{10}(t)+ \\
& q_{8,11}^{(10)}(t) @ B_{11}(t)+q_{8,13}(t) @ B_{13}(t) \\
& B_{9}(t)=M_{9}+q_{95}(t) \Subset B_{5}(t) \\
& B_{10}(t)=M_{10}+q_{10,7}(t) \subseteq B_{7}(t)+q_{10,11}(t) \subseteq B_{11}(t) \\
& B_{11}(t)=M_{11}+q_{11,10}(t) @ B_{10}(t) \\
& B_{12}(t)=M_{12}+q_{12,7}(t) \subseteq B_{7}(t) \\
& B_{13}(t)=M_{13}+q_{13,8}(t) \subseteq B_{8}(t)
\end{aligned}
$$

Taking the LT of above equations and solving for $B_{0}^{*}(s)$,

$$
B_{0}^{*}(s)=\frac{N_{3}(s)}{D_{3}(s)}
$$

In steady state, BPA is given by

$$
\begin{aligned}
& B_{0}=\lim _{s \rightarrow 0} s B_{0}^{*}(s) \frac{N_{3}(0)}{D_{3}^{\prime}(0)} \\
& N_{3}(0)=\phi_{8} p_{58} p_{02} p_{25} p_{32} p_{75} p_{10,7}+\left[\phi _ { 7 } \left\{p_{57}(1-\right.\right. \\
& \left.\left.\left.p_{8,13}\right)+p_{58} p_{87}^{(10)}\right\}\right] p_{02} p_{25} p_{32} p_{10,7}+\left[\phi_{10}(1-\right. \\
& \left.p_{7,12}\right)\left\{\left(1-p_{8,13}\right)\left(1-p_{59}\right)-p_{58} p_{85}\right\}-p_{75}\left\{p_{57}(1-\right. \\
& \left.\left.\left.p_{8,13}\right)+p_{58} p_{87}^{(10)}\right\}\right] p_{02} p_{32} p_{25}+\phi_{11}\left[p_{10,11}(1-\right. \\
& \left.p_{7,12}\right)\left\{\left(1-p_{8,13}\right)\left(1-p_{59}\right)-p_{58} p_{85}\right\} p_{75} p_{10,11}\left\{p_{57}(1-\right. \\
& \left.\left.\left.p_{8,13}\right)+p_{58} p_{87}^{(10)}\right\}\right] p_{02} p_{32} p_{25}+\left[\phi _ { 1 2 } \left\{\left(1-p_{8,13}\right)(1-\right.\right. \\
& \left.\left.\left.p_{59}\right)-p_{58} p_{85}\right\} p_{7,12}+\phi_{13} p_{75} p_{8,13} p_{58}\right] p_{02} p_{25} p_{32} p_{10,7}
\end{aligned}
$$

And $D_{3}^{\prime}(0)=D_{2}^{\prime}(0)$ given by $(2)$

\section{7- Expected No. of Visits by Repairman}

We denote $V_{i}(t)$ as the expected no. of visits by the repairperson to repair the failed unit. The expected no of visits for different states is given as:

$$
\begin{aligned}
V_{0}(t)= & q_{01}(t) \Subset V_{1}(t)+q_{02}(t) \Subset V_{2}(t) \\
V_{1}(t)= & q_{10}(t) \Subset V_{0}(t) \\
V_{2}(t)= & q_{23}(t) \Subset V_{3}(t)+q_{24}(t)\left[1+V_{4}(t)\right]+ \\
& q_{25}(t) \Subset V_{5}(t) \\
V_{3}(t)= & q_{32}(t) \Subset V_{2}(t)+q_{36}(t) \Subset V_{6}(t)
\end{aligned}
$$

$$
\begin{aligned}
& V_{4}(t)=q_{42}(t)\left(V_{2}(t)+q_{43}(t) \Subset V_{3}(t)\right. \\
& V_{5}(t)=q_{57}(t)\left[1+V_{7}(t)\right]+q_{58}(t)\left[1+V_{8}(t)\right]+ \\
& q_{59}(t)\left[1+V_{9}(t)\right] \\
& V_{6}(t)=q_{63}(t) @ V_{3}(t) \\
& V_{7}(t)=q_{75}(t)\left(V_{5}(t)+q_{7,10}(t)(C) V_{10}(t)+\right. \\
& q_{7,12}(t) @ V_{12}(t) \\
& V_{8}(t)=q_{85}(t) \text { (C) } V_{5}(t)+q_{8,13}(t) \text { (c) } V_{13}(t)+ \\
& q_{87}^{(10)}(t) \Subset V_{7}(t)+q_{8,11}^{(10)}(t) \Subset V_{11}(t) \\
& V_{9}(t)=q_{95}(t) \subset V_{5}(t) \\
& V_{10}(t)=q_{10,7}(t) \text { (C) } V_{7}(t)+q_{10,11}(t)\left(C V_{11}(t)\right. \\
& V_{11}(t)=q_{11,10}(t)\left(V_{10}(t)\right. \\
& V_{12}(t)=q_{12,7}(t) \Subset V_{7}(t) \\
& V_{13}(t)=q_{13,8}(t) \Subset V_{8}(t)
\end{aligned}
$$

Taking the $\mathrm{LT}$ of above equations and solving for $V_{0}^{*}(s)$,

$$
V_{0}^{*}(s)=\frac{N_{4}(s)}{D_{4}(s)}
$$

In steady state, the no. of visits per unit time is given by

$$
\mathrm{V}_{0}(0)=\lim _{\mathrm{s} \rightarrow 0} \mathrm{sV}_{0}^{*}(\mathrm{~s})=N_{3}(0) / D_{4}^{\prime}(0)
$$

Where,

$$
\begin{aligned}
& N_{4}(0)=\left[p_{01}\left\{\left(1-p_{24} p_{42}\right) p_{32}-\left\{p_{23}+p_{24} p_{43}^{(6)}\right\} p_{32}\right\}+\right. \\
& \left.p_{02}\left(p_{23}+p_{24}+p_{25}\right) p_{32}\right]\left[p _ { 1 0 , 7 } p _ { 7 , 5 } \left(1-p_{85} p_{58}-\right.\right. \\
& \left.\left.p_{59}\left(1-p_{8,13}\right)\right)\right]-\left\{p_{57}\left(1-p_{8,13}\right)+p_{58} p_{87}^{(10)}\right\} p_{75} p_{10,11} \\
& +\left\{p_{02} p_{25} p_{32}\left(p_{57}+p_{58}+p_{59}\right)\left(1-p_{8,13}\right)\right\} p_{10,7} p_{75}
\end{aligned}
$$

And $D_{4}^{\prime}(0)=D_{2}^{\prime}(0)$ given in (2)

\section{8- Profit Analysis}

The profit encountered to the model in steady state by considering different costs and revenues, is calculated as:

Where

$$
P_{1}=K_{0} A_{0}-K_{1} B_{0}-K_{2} V_{0}
$$

$\mathrm{K}_{0}=$ Revenue per unit up time of the system,

$\mathrm{K}_{1}=$ Cost per unit time for which the repair is busy, $\mathrm{K}_{2}=$ Cost per unit visits by the repairman. 


\section{9- Behavior of Reliability Measures:}

In this section, for practical illustration, the behavior of some important reliability measures has been examined for some specific values of parameters.

Table 1.Performance of $\eta_{1}$ and fixed parameters $\eta_{2}, \eta_{3}, \delta_{1}, \delta_{2}, \alpha_{1}$,

\begin{tabular}{|c|c|c|c|}
\hline \multirow[b]{2}{*}{$\eta_{1}$} & \multicolumn{3}{|l|}{ MTSF } \\
\hline & $\begin{array}{l}\eta_{2}=0.10 \\
\eta_{3}=0.04 \\
\delta_{1}=0.74 \\
\delta_{2}=0.60 \\
\alpha_{1}=0.47 \\
\alpha_{2}=0.24 \\
\alpha_{3}=0.33\end{array}$ & $\begin{array}{l}\eta_{2}=0.08 \\
\eta_{3}=0.02 \\
\delta_{1}=0.79 \\
\delta_{2}=0.69 \\
\alpha_{1}=0.34 \\
\alpha_{2}=0.39 \\
\alpha_{3}=0.37\end{array}$ & $\begin{array}{l}\eta_{2}=0.15 \\
\eta_{3}=0.06 \\
\delta_{1}=0.64 \\
\delta_{2}=0.50 \\
\alpha_{1}=0.37 \\
\alpha_{2}=0.34 \\
\alpha_{3}=0.39\end{array}$ \\
\hline 0.1 & 3.9904 & 5.2570 & 2.8029 \\
\hline 0.2 & 2.9559 & 3.9690 & 2.3256 \\
\hline 0.3 & 2.3585 & 3.2605 & 2.0005 \\
\hline 0.4 & 1.9677 & 2.8004 & 1.7637 \\
\hline 0.5 & 1.6917 & 2.4719 & 1.5834 \\
\hline 0.6 & 1.4863 & 2.2227 & 1.4416 \\
\hline 0.7 & 1.3276 & 2.0256 & 1.3271 \\
\hline 0.8 & 1.2013 & 1.8648 & 1.2328 \\
\hline 0.9 & 1.0985 & 1.7307 & 1.1537 \\
\hline 1.0 & 1.0065 & 1.6612 & 1.0653 \\
\hline
\end{tabular}

Table 2. Performance of $\alpha_{1}$ and fixed parameters $\eta_{2}, \eta_{3}, \delta_{1}, \delta_{2}, \eta_{1}$, $\alpha_{2}$ and $\alpha_{3}$ on MTSF.

\begin{tabular}{|l|l|l|l|}
\hline \multirow{2}{*}{$\alpha_{1}$} & \multicolumn{3}{|l}{ MTSF } \\
\cline { 2 - 4 } & $\eta_{2}=0.13$ & $\eta_{2}=0.15$ & $\eta_{2}=0.11$ \\
& $\eta_{3}=0.25$ & $\eta_{3}=0.18$ & $\eta_{3}=0.21$ \\
& $\delta_{1}=0.31$ & $\delta_{1}=0.34$ & $\delta_{1}=0.31$ \\
& $\delta_{2}=0.33$ & $\delta_{2}=0.43$ & $\delta_{2}=0.33$ \\
& $\eta_{1}=0.27$ & $\eta_{1}=0.29$ & $\eta_{1}=0.23$ \\
& $\alpha_{2}=0.03$ & $\alpha_{2}=0.04$ & $\alpha_{2}=0.02$ \\
& $\alpha_{3}=0.33$ & $\alpha_{3}=0.33$ & $\alpha_{3}=0.33$ \\
\hline 0.1 & 1.8571 & 1.6701 & 2.1038 \\
\hline 0.2 & 2.0716 & 1.8292 & 2.4664 \\
\hline 0.3 & 2.1878 & 1.9227 & 2.6476 \\
\hline 0.4 & 2.2646 & 1.9869 & 2.7667 \\
\hline 0.5 & 2.3221 & 2.0350 & 2.8596 \\
\hline 0.6 & 2.3680 & 2.0727 & 2.9397 \\
\hline 0.7 & 2.4054 & 2.1019 & 3.0123 \\
\hline 0.8 & 2.4351 & 2.1230 & 3.0792 \\
\hline 0.9 & 2.4563 & 2.1352 & 3.1401 \\
\hline 1.0 & 2.4677 & 2.1369 & 3.1938 \\
\hline
\end{tabular}

Table 3. Performance of $\eta_{1}$ and fixed parameters $\eta_{2}, \eta_{3}, \delta_{1}, \delta_{2}, \alpha_{1}, \alpha_{2}$, $\alpha_{3}, k_{0}, k_{1}$ and $k_{2}$ on Profit.

\begin{tabular}{|c|c|c|c|}
\hline \multirow{2}{*}{$\eta_{1}$} & \multicolumn{3}{|l|}{ Profit } \\
\cline { 2 - 4 } & $\eta_{2}=0.81$ & $\eta_{2}=0.79$ & $\eta_{2}=0.89$ \\
& $\eta_{3}=0.95$ & $\eta_{3}=0.93$ & $\eta_{3}=0.97$ \\
& $\delta_{1}=0.91$ & $\delta_{1}=0.91$ & $\delta_{1}=0.81$ \\
& $\delta_{2}=0.39$ & $\delta_{2}=0.39$ & $\delta_{2}=0.32$ \\
& $\alpha_{1}=0.56$ & $\alpha_{1}=0.55$ & $\alpha_{1}=0.54$ \\
& $\alpha_{2}=0.32$ & $\alpha_{2}=0.35$ & $\alpha_{2}=0.33$ \\
& $\alpha_{3}=0.33$ & $\alpha_{3}=0.33$ & $\alpha_{3}=0.33$ \\
& $k_{0}=1000$ & $k_{0}=1000$ & $k_{0}=950$ \\
& $k_{1}=100$ & $k_{1}=120$ & $k_{1}=120$ \\
& $k_{2}=50$ & $k_{2}=90$ & $k_{2}=90$ \\
\hline 0.1 & 2.5482 & 2.4729 & 2.8455 \\
\hline 0.2 & 2.1945 & 2.2126 & 2.4681 \\
\hline 0.3 & 1.8936 & 2.0004 & 2.1495 \\
\hline 0.4 & 1.6221 & 1.8157 & 1.8639 \\
\hline 0.5 & 1.3656 & 1.6459 & 1.5954 \\
\hline 0.6 & 1.1151 & 1.4828 & 1.3339 \\
\hline 0.7 & 0.8643 & 1.3212 & 1.0724 \\
\hline 0.8 & 0.6091 & 1.1574 & 0.8061 \\
\hline 0.9 & 0.3463 & 0.9888 & 0.5318 \\
\hline 1.0 & 0.0737 & 0.8134 & 0.2468 \\
\hline & & & \\
\hline
\end{tabular}

Table 4. Performance of $\alpha_{1}$ and fixed parameters $\eta_{2}, \eta_{3}, \delta_{1}, \delta_{2}, \eta_{1}, \alpha_{2}$, $\alpha_{3}, k_{0}, k_{1}$ and $k_{2}$ on Profit.

\begin{tabular}{|l|l|l|l|}
\hline \multirow{2}{*}{$\alpha_{1}$} & \multicolumn{3}{|l|}{ Profit } \\
\cline { 2 - 4 } & $\eta_{2}=0.65$ & $\eta_{2}=0.69$ & $\eta_{2}=0.61$ \\
& $\eta_{3}=0.59$ & $\eta_{3}=0.62$ & $\eta_{3}=0.55$ \\
& $\delta_{1}=0.65$ & $\delta_{1}=0.55$ & $\delta_{1}=0.54$ \\
& $\delta_{2}=0.49$ & $\delta_{2}=0.49$ & $\delta_{2}=0.39$ \\
& $\eta_{1}=0.47$ & $\eta_{1}=0.45$ & $\eta_{1}=0.35$ \\
& $\alpha_{2}=0.69$ & $\alpha_{2}=0.72$ & $\alpha_{2}=0.75$ \\
& $\alpha_{3}=0.55$ & $\alpha_{3}=0.56$ & $\alpha_{3}=0.59$ \\
& $k_{0}=950$ & $k_{0}=900$ & $k_{0}=1000$ \\
& $k_{1}=120$ & $k_{1}=150$ & $k_{1}=100$ \\
& $k_{2}=90$ & $k_{2}=80$ & $k_{2}=50$ \\
\hline 0.1 & 3.59827 & 4.17309 & 3.80843 \\
\hline 0.2 & 3.67789 & 4.37047 & 3.85116 \\
\hline 0.3 & 3.83764 & 4.68783 & 3.99023 \\
\hline 0.4 & 4.08536 & 5.15737 & 4.23971 \\
\hline 0.5 & 4.44261 & 5.84607 & 4.63524 \\
\hline 0.6 & 4.95729 & 6.89995 & 5.2584 \\
\hline 0.7 & 5.739 & 8.68624 & 6.31344 \\
\hline 0.8 & 7.08058 & 12.4277 & 8.45213 \\
\hline 0.9 & 10.0689 & 25.9994 & 15.3405 \\
\hline 1.0 & 11.1245 & 30.3421 & 20.4563 \\
\hline & & & \\
\hline
\end{tabular}

From Table 1, it has been observed that as failure rate " $\eta_{1}$ " increases, keeping the other parameters $\eta_{2}, \eta_{3}, \delta_{1}$, 
$\delta_{2}, \alpha_{1}, \alpha_{2}$ and $\alpha_{3}$ fixed, mean time to system failure decreases. This implies that expected life of the system decreases as failure rate increases. Thus we conclude that mean time to system failure can be increased by reducing failure rate. From Table 2, it has been seen that as repair rate " $\alpha_{1}$ " increases, keeping the values of other parameters $\eta_{2}, \eta_{3}, \delta_{1}, \delta_{2}, \alpha_{1}, \eta_{1}$ and $\alpha_{3}$ fixed, the mean time to system failure increases. This implies that expected life of the system increases with increasing repair rate. Further, from Table 3, it has been observed that profit of the model decreases as failure rate " $\eta_{1}$ " increases, irrespective of the other parameters $\eta_{2}, \eta_{3}, \delta_{1}, \delta_{2}, \alpha_{1}, \alpha_{2}, \alpha_{3}, \mathrm{k}_{0}, \mathrm{k}_{1}$ and $\mathrm{k}_{2}$. Hence it can be concluded that expected life of the system can be increased by reducing failure rate. Also from Table 4 , it has been noticed that the profit of the model increases as repair rate " $\alpha_{1}$ " increases, keeping the other parameters $\eta_{2}, \eta_{3}, \delta_{1}, \delta_{2}, \alpha_{1}, \eta_{1}$ and $\alpha_{3}$ fixed. This implies that expected life of the system increases as repair rate increases.

\section{0- Conclusion}

In this paper, the profit analysis of the model comprising of three non-identical units has been obtained. The reliability technique of priority of one unit over other has been used. Expressions for various important reliability measures have been obtained using regenerative point technique. The numerical illustration reveals that MTSF and profit of the system decreases with increase in the failure rate. Further MTSF and profit of the model increases as repair rate increases. Thus we conclude that expected lifetime of the model can be enriched by either increasing the repair rate of the unit or by reducing the failure rate of the unit which in turn will enhance the performance and hence efficiency of the model. The study advocates that some preventive measures should be taken to avoid failure of the system, for that the priority strategy of reliability measure should be taken into consideration.

The results obtained in this research paper are useful for system planners who are engaged in designing highly reliable system. This is significant because it considers an actual problem and provides a unique conceptual model as a starting point for organizations to foster higher levels of engagement and check the efficiency and effectiveness of the system. It is Novel Research and good topic for research for manufacturers, designers and users.

\section{References}

[1] A. Kumar and S. C. Malik, "Reliability Analysis of a Redundant System with 'FCFS' Repair Policy Subject to Weather Conditions", International Journal of Advanced Science and Technology, Vol. 29, No. 03, 2020, pp. $7568-$ 7578.

[2] A. Kumar, D. Pawar, and S. C. Malik, "Profit analysis of a warm standby non-identical unit system with single server performing in normal/abnormal environment," Life Cycle Reliab Saf Eng., doi 10.1007/s41872-019-00083-2. 2019.

[3] Chib, Rakesh, J.P. S. Joorel, and V. Sharma "Profit Analysis of a Two Unit Priority System with Two Types of Repair Facility", International Journal of Statistics and Reliability Engineering, Vol. 1, No. 1, 2014, pp. 69-81

[4] I. kumar, A. Kumar, and M. Saina, "Analysis of performance measures of computer systems with priority and maximum operation time," Advances in intelligent systems and computing,Springer Singapore, vol 933, 2019.

[5] J.K. Sureria and S.C. Malik "Reliability Measures of a Computer System with Arrival Time of the Server and Priority to H/w Repair over S/w Up-gradation", International Journal of Computer Applications, Vol. 65, No.13, 2013, pp. 44-48.

[6] M.S. Kadyan, S.C.Malik and J.Kumar, "Cost-Analysis of a System under Priority to Repair and Degradation" International Journal of Statistics and Systems, Vol. 5, No. 1, 2010, pp. 1-10.

[7] N. Bashir, R. Bashir and T R Jan, "Effectiveness Analysis of Two Non-Identical Unit System Model with Priority Unit Subject to Degradation and Inspection Facility", IEEE International Conference on Computational Intelligence and Knowledge Economy (ICCIKE), 2019, pp. 40-45. ISBN 9781-7281-3778-0

[8] N. Gorjian Jolfaei , B. Jin , L. van der Linden , I. Gunawan and N. Gorjian, "Reliability modelling with redundancy-A case study of power generation engines in a wastewater treatment plant", Qual Reliab Engng Int. 2019, pp. 1-13. https://doi.org/10.1002/qre.2573

[9] R. Rathee and S. Chander "A Parallel System with Priority to Repair over Preventive Maintenance Subject to Maximum Operation and Repair Times", International Journal of Statistics and Reliability Engineering, Vol. 1, No. 1, 2014, pp. 57-68.

[10] R. Sharma and J.P.S. Joorel, "Reliability modelling and cost benefit analysis of fruit juice manufacturing system". Mathematics in Engineering, Science and Aerospace, Vol. 6, No. 2, 2015, pp. 281-293.

[11] S.Chander "Reliability models with priority for operation and repair with arrival time of server", Pure and Applied Mathematika Sciences, Vol. XI, No. 1-2, 2005, pp. 9-22.

[12] S.C.Malik, M.S. Kadian, and J. Kumar " "Cost-Analysis of a System under Priority to Repair and Degradation", International Journal of Statistics and Systems, Vol. 5,No.1, 2010, pp. $1-10$.

[13] S.C. Malik, "Reliability Modeling of a computer System with Preventive Maintenance and Priority Subject to Maximum Operation and Repair Times", International Journal of System Assurance Engineering and Management, Vol. 4(1), 2013, pp. 94-100. 
[14] V.J. Munday and S.C. Malik, "Reliability Measures of a Computer System with Priority for Repair and Hardware Redundancy" , International Journal of Computer Applications, Vol. 125, No.8, 2015, pp. 32-37.

[15] Y.L.Zhang and G.J. Wang, "A Geometric Process Repair Model for a Repairable Cold Standby System with Priority in Use and Repair", Journal of Reliability Engineering and System Safety, Vol. 94, 2009, pp. 90-100.

[16] M.S. Barak , Neeraj, and S.K. Barak "Proft analysis of a two-unit cold standby system model operating under different weather conditions", Life Cycle Reliability and Safety Engineering, Springer, 2018, https://doi.org/10.1007/s4187 2-018-0048-6

[17] O. Dahiya, A. Kumar, and M. Saini, "Modeling and analysis of concrete mixture plant subject to coverage factor and profust reliability approach", Life Cycle Reliability and Safety Engineering, Springer, 9, 2020, pp. 273-281, https://doi.org/10.1007/s41872-019-00104-0

[18] D. Goyal, A. Kumar, M. Saini, and H. Joshi. "Reliability, maintainability and sensitivity analysis of physical processing unit of sewage treatment plant", SN Applied Sciences, Springer, 2020. https://doi.org/10.1007/s42452019-1544-7

[19] N. Gupta, M. Saini, and A. Kumar, "Operational availability analysis of generators in steam turbine power plants" SN Applied Sciences, Springer, 2020. https://doi.org/10.1007/s42452-020-2520-y

Ms. Nafeesa Bashir received Masters degree in Statistics from University of Kashmir, J\&K, India. Currently she is a Ph.D. research Scholar in University of Kashmir. She has to her credit several research publications both in national and international journals of repute. Her research interest include Reliability Modeling and Probability Distributions.

Dr. Raeesa Bashir (Ph.D. Statistics) from University of Jammu, India. She is an Assistant Professor, in Mathematics and Quantitative Analysis in the Engineering and Management Department at Amity University Dubai with many years of experience in teaching and research. She has to her credit several articles and research papers in reputed journals. She has previously worked in government and private organizations in Kingdom of Saudi Arabia and UAE.

Prof J P Singh Joorel is a Professor of Statistics in the Faculty of Mathematical Sciences at University of Jammu, Jammu, India and presently heading the INFLIBNET Centre (An Autonomous Inter University Centre of University Grants Commission, Govt of India), Gandhinagar, Gujarat as Director. He received his Doctorate degree in Statistics from Institute of Social Sciences, Agra University, Agra, India in 1989. He has published work in international and national journals of repute. His areas of interest are Reliability Theory, Sample Surveys and Statistical Inference.

Dr. Tariq Rashid Jan is an Associate Professor in the Department of Statistics, University of Kashmir, J\&K, India. He did his M. Phil. in Statistics from Department of Statistics and Operations Research, Aligarh Muslim University, Aligarh, India. He received his $\mathrm{Ph}$. D. degree in Statistics from
Department of Statistics, University of Kashmir, Srinagar, India in 2004. He has published work in international and national journals of repute. His current research interests are in Reliability Theory, Bio -Statistics and Generalized Distributions. 